\title{
THE PTF ORION PROJECT: A POSSIBLE PLANET TRANSITING A T-TAURI STAR
}

\author{
Julian C. van Eyken ${ }^{1,17}$, David R. Ciardi ${ }^{1,17}$, Kaspar von Braun ${ }^{1}$, Stephen R. Kane $^{1}$, Peter Plavchan $^{1}$, \\ Chad F. Bender ${ }^{2,3}$, Timothy M. Brown ${ }^{4}$, Justin R. Crepp ${ }^{5}$, Benjamin J. Fulton ${ }^{4}$, Andrew W. Howard ${ }^{6}$, \\ Steve B. Howell ${ }^{7,17}$, Suvrath Mahadevan ${ }^{2,3}$, Geoffrey W. Marcy ${ }^{6}$, Avi Shrorer ${ }^{4}$, Paula SzKody ${ }^{8,17}$, \\ Rachel L. Akeson $^{1}$, Charles A. Beichman ${ }^{1}$, Andrew F. Boden ${ }^{9}$, Dawn M. Gelino ${ }^{1}$, D. W. Hoard ${ }^{10}$, \\ Solange V. Ramírez ${ }^{1}$, Luisa M. Rebull ${ }^{10}$, John R. Stauffer ${ }^{10}$, Joshua S. Bloom ${ }^{6}$, S. Bradley Cenko 6 , \\ Mansi M. Kasliwal ${ }^{5}$, Shrinivas R. Kulkarni ${ }^{5}$, Nicholas M. Law ${ }^{11}$, Peter E. Nugent ${ }^{12}$, Eran O. OfeK ${ }^{13,18}$, \\ Dovi Poznanski ${ }^{14}$, Robert M. Quimby ${ }^{15}$, Richard Walters ${ }^{5}$, Carl J. Grillmair ${ }^{10}$, Russ Laher ${ }^{10}$, \\ David B. Levitan ${ }^{16}$, Branimir Sesar ${ }^{5}$, and Jason A. Surace ${ }^{10}$ \\ ${ }^{1}$ NASA Exoplanet Science Institute, California Institute of Technology, 770 South Wilson Avenue, M/S 100-22, Pasadena, \\ CA 91125, USA; vaneyken@ipac.caltech.edu \\ ${ }^{2}$ Department of Astronomy \& Astrophysics, The Pennsylvania State University, University Park, PA 16802, USA \\ ${ }^{3}$ Center for Exoplanets and Habitable Worlds, The Pennsylvania State University, University Park, PA 16802, USA \\ ${ }^{4}$ Las Cumbres Observatory Global Telescope, Goleta, CA 93117, USA \\ ${ }^{5}$ Cahill Center for Astrophysics, California Institute of Technology, Pasadena, CA 91125, USA \\ ${ }^{6}$ Department of Astronomy, University of California, Berkeley, CA 94720-3411, USA \\ 7 NASA Ames Research Center, M/S 244-30, Moffett Field, CA 94035, USA \\ ${ }^{8}$ Department of Astronomy, University of Washington, Box 351580, Seattle, WA 98195, USA \\ ${ }^{9}$ Caltech Optical Observatories, California Institute of Technology, Pasadena, CA 91125, USA \\ ${ }^{10}$ Spitzer Science Center, M/S 220-6, California Institute of Technology, Jet Propulsion Laboratory, Pasadena, CA 91125, USA \\ ${ }^{11}$ Dunlap Institute for Astronomy and Astrophysics, University of Toronto, 50 St. George Street, Toronto M5S 3H4, Ontario, Canada \\ ${ }^{12}$ Computational Cosmology Center, Lawrence Berkeley National Laboratory, 1 Cyclotron Road, Berkeley, CA 94720, USA \\ ${ }^{13}$ Benoziyo Center for Astrophysics, Weizmann Institute of Science, 76100 Rehovot, Israel \\ ${ }^{14}$ School of Physics and Astronomy, Tel Aviv University, Tel Aviv 69978, Israel \\ ${ }^{15}$ IPMU, University of Tokyo, Kashiwanoha 5-1-5, Kashiwa-shi, Chiba, Japan \\ ${ }^{16}$ Division of Physics, Mathematics and Astronomy, California Institute of Technology, Pasadena, CA 91125, USA \\ Received 2011 October 24; accepted 2012 June 6; published 2012 July 25
}

\begin{abstract}
We report observations of a possible young transiting planet orbiting a previously known weak-lined T-Tauri star in the 7-10 Myr old Orion-OB1a/25-Ori region. The candidate was found as part of the Palomar Transient Factory (PTF) Orion project. It has a photometric transit period of $0.448413 \pm 0.000040$ days, and appears in both 2009 and 2010 PTF data. Follow-up low-precision radial velocity (RV) observations and adaptive optics imaging suggest that the star is not an eclipsing binary, and that it is unlikely that a background source is blended with the target and mimicking the observed transit. RV observations with the Hobby-Eberly and Keck telescopes yield an RV that has the same period as the photometric event, but is offset in phase from the transit center by $\approx-0.22$ periods. The amplitude (half range) of the RV variations is $2.4 \mathrm{~km} \mathrm{~s}^{-1}$ and is comparable with the expected RV amplitude that stellar spots could induce. The RV curve is likely dominated by stellar spot modulation and provides an upper limit to the projected companion mass of $M_{\mathrm{p}} \sin i_{\text {orb }} \lesssim 4.8 \pm 1.2 M_{\text {Jup }}$; when combined with the orbital inclination, $i_{\text {orb }}$, of the candidate planet from modeling of the transit light curve, we find an upper limit on the mass of the planetary candidate of $M_{\mathrm{p}} \lesssim 5.5 \pm 1.4 M_{\text {Jup }}$. This limit implies that the planet is orbiting close to, if not inside, its Roche limiting orbital radius, so that it may be undergoing active mass loss and evaporation.
\end{abstract}

Key words: open clusters and associations: individual (25 Ori) - planets and satellites: detection - stars: individual (2MASS J05250755+0134243, CVSO 30, PTFO 8-8695, PTF1 J052507.55+013424.3) - stars: pre-main sequence

Online-only material: color figures

\section{INTRODUCTION}

The Palomar Transient Factory (PTF) Orion project is a study within the broader PTF survey aimed at searching for photometric variability in the young Orion region, with the primary goal of finding young extrasolar planets (van Eyken et al. 2011). The project is based on a set of intensive highcadence $(\approx 70-90 \mathrm{~s})$ observations of a single $7.26 \mathrm{deg}^{2}$ field centered on the known 25 Ori association, which lies in the Orion

\footnotetext{
${ }^{17}$ Visiting Astronomer, Kitt Peak National Observatory, National Optical Astronomy Observatory, which is operated by the Association of Universities for Research in Astronomy, Inc. (AURA), under cooperative agreement with the National Science Foundation.

${ }^{18}$ Einstein Fellow.
}

OB1a region and has an estimated age of 7-10 Myr (Briceño et al. 2005, 2007).

Typical young circumstellar disk lifetimes are on the order of 5-10 Myr (Hillenbrand 2008), and it is during this time that the bulk of the formation and migration of planets is expected to occur. The youngest exoplanets have been found via direct detection (e.g., LkCa 15, Kraus \& Ireland 2012; the free-floating planetary-mass object in the $\rho$ Oph cloud, Marsh et al. 2010); however, little is known empirically about exoplanets during the first few millions of years of their lives, and the goal is to fill the observational gap and begin to provide constraints on theories of planet formation and evolution (see, e.g., Armitage 2009). The stars in the 25 Ori/OB1a region should be at or just beyond the point of disk dissipation, consistent with the high ratio of 
Table 1

PTFO 8-8695 Stellar Properties

\begin{tabular}{|c|c|}
\hline Property & Value \\
\hline \multirow[t]{3}{*}{ Alternative designations } & CVSO 30 \\
\hline & 2MASS J05250755+0134243 \\
\hline & PTF1 J052507.55+013424.3 \\
\hline$\alpha(\mathrm{J} 2000)$ & $05^{\mathrm{h}} \cdot 25^{\mathrm{m}} \cdot 07^{\mathrm{s}} .55$ \\
\hline$\delta(\mathrm{J} 2000)$ & $+01^{\circ} .34^{\prime} .24 .3^{\prime \prime}$ \\
\hline$V$ & $16.26 \mathrm{mag}^{\mathrm{a}}$ \\
\hline 2MASS $J$ & $12.232 \pm 0.028 \mathrm{mag}^{\mathrm{b}}$ \\
\hline 2MASS $H$ & $11.559 \pm 0.026 \mathrm{mag}^{\mathrm{b}}$ \\
\hline $2 \mathrm{MASS} K_{S}$ & $11.357 \pm 0.021 \mathrm{mag}^{\mathrm{b}}$ \\
\hline Median $R$ & $15.19 \mathrm{mag}^{\mathrm{c}}$ \\
\hline$R$ range & $0.17 \mathrm{mag}(\min \text { to } \max )^{\mathrm{c}}$ \\
\hline $\mathrm{H}_{\alpha}$ equivalent width & $-11.40 \AA^{\mathrm{a}}$ \\
\hline LiI equivalent width & $0.40 \AA^{\mathrm{a}}$ \\
\hline Sp. Type & M3 (PMS weak-lined T-Tauri) ${ }^{\mathrm{a}}$ \\
\hline$T_{\text {eff }}$ & $3470 \mathrm{~K}^{\mathrm{a}}$ \\
\hline$A_{V}$ & $0.12 \mathrm{mag}^{\mathrm{a}}$ \\
\hline Luminosity & $0.25 L_{\odot}^{\mathrm{a}}$ \\
\hline Radius & $1.39 R_{\odot}^{\mathrm{a}, \mathrm{d}}$ \\
\hline Mass (Baraffe/Siess) & $0.44 M_{\odot} / 0.34 M_{\odot}{ }^{\mathrm{a}, \mathrm{e}}$ \\
\hline Age (Baraffe/Siess) & 2.63 Myr/2.68 $\mathrm{Myr}^{\mathrm{a}, \mathrm{e}}$ \\
\hline Distance & $\sim 330 \mathrm{pc}$ (mean dist. to OB1a/25 Ori assoc.) $)^{\mathrm{a}, \mathrm{f}}$ \\
\hline
\end{tabular}

Notes.

a Briceño et al. (2005).

b Skrutskie et al. (2006).

c From PTF Orion data (this paper).

d cf., smaller value implied by PTF Orion transit measurements-see Table 3.

e Reported by Briceño et al. (2005) based on comparison with Baraffe et al. (1998) and Siess et al. (2000) stellar models.

${ }^{\mathrm{f}}$ Briceño et al. (2007).

weak-lined to classical T-Tauri stars found there (Briceño et al. 2007). We can therefore look for planet transits at the time when they may first become observable without their signatures being swamped by the extreme variability characteristic of the younger classical T-Tauri stars (CTTSs). In so doing, we can begin to investigate the frequency of planets at these ages, the timescales for their evolution, the timescales of their migration with respect to the star's evolution, and, through measurements of the transit depths, probe empirically their mean densities and the extent of their atmospheres, which are expected to be inflated at these early stages (Fortney \& Nettelmann 2010 and references therein).

Several surveys have been undertaken to search for closein young exoplanets, though many are radial velocity (RV) searches (e.g., Esposito et al. 2006; Paulson \& Yelda 2006; Setiawan et al. 2007, 2008; Huerta et al. 2008; Crockett et al. 2011; Nguyen et al. 2012). A transit search (e.g., Aigrain et al. 2007; Miller et al. 2008; Neuhäuser et al. 2011) has the advantage of being able to search many more stars simultaneously, down to fainter magnitudes and with a faster cadence. Combining transit photometry with spectroscopic information can provide a wealth of information that is unavailable with non-transiting planets, from masses and radii to constraints on atmospheric composition.

An outline of the PTF Orion project is given by van Eyken et al. (2011), along with some of the first results concerning binary stars and T-Tauri stars (TTSs). The broader PTF survey is described in detail by Law et al. (2009) and Rau et al. (2009), with a summary of the first year's performance by Law et al. (2010). Here, we report a young planet candidate found orbiting a known M3 pre-main-sequence (PMS) weak-

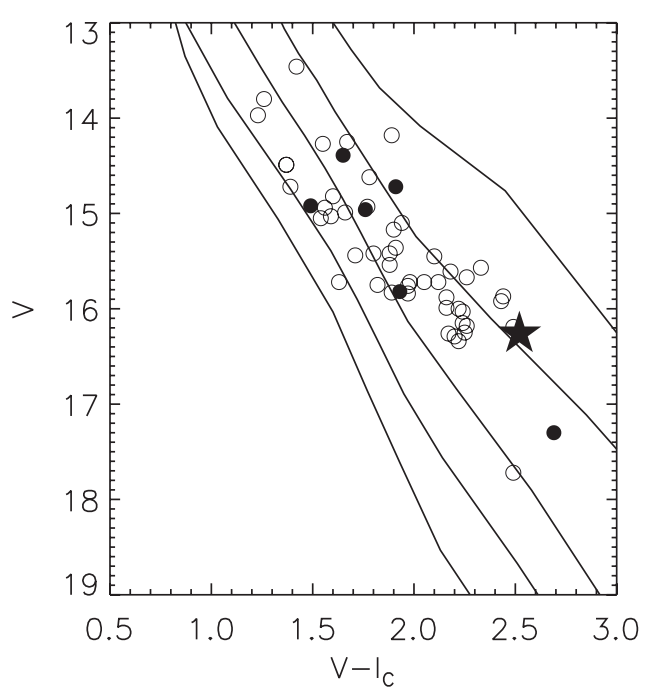

Figure 1. Color-magnitude diagram following Briceño et al. (2005, Figure 7), highlighting PTFO 8-8695 (CVSO 30) - the star symbol-in relation to the other T-Tauri stars discovered therein (see Section 2.1). PTFO 8-8695 lies at the younger end of the distribution. Open circles indicate WTTS; filled circles indicate CTTS. Solid lines indicate, from the top, 1, 3, 10, and $30 \mathrm{Myr}$ isochrones, and the zero-age main sequence, according to the models of Siess et al. (2000) at a distance of $330 \mathrm{pc}$. Photometric measurements are reproduced from Briceño et al. (2005). Reddening and extinction are neglected.

lined T-Tauri star (WTTS) within the PTF Orion field. Our observations are outlined in Section 2; Section 3 discusses the photometric and spectroscopic results and some implications. The main conclusions are summarized in Section 4.

\section{OBSERVATIONS}

\subsection{PTF Photometry}

PTF Orion data were obtained using the Palomar 48" Samuel Oschin telescope during the majority of the clear nights between 2009 December 1 and 2010 January 15, whenever the field was above an air mass of 2.0. All observations were in the $R$ band, and of the 40 nights dedicated, 14 yielded usable data, the remainder being lost primarily due to poor weather. Light curves were obtained for $\sim 110,000$ sources within the field, the top $\sim 500$ most variable of which were inspected visually. The observations and the PTF Orion differential photometry pipeline and data reduction details are described fully in van Eyken et al. (2011). Specifics of the more general PTF data reduction pipeline are described by R. Laher et al. (2012, in preparation) and Ofek et al. (2012). Among the inspected sources, one, PTFO 8-8695 (2MASS J05250755+0134243), showed periodic shallow transit-like events with a shape and depth suggestive of a planetary companion, superposed on a larger-scale quasiperiodic variable light curve. The source has previously been identified as a WTTS associated with the Orion OB1a region by Briceño et al. (CVSO 30, 2005) and Hernández et al. (2007). A summary of the main previously determined properties of the primary star is given in Table 1 . In Figure 1, we show a color-magnitude diagram indicating PTFO 8-8695 in relation to the other TTSs in the OB1 a region discovered by Briceño et al. (2005). Although classified as a WTTS, it lies at the younger end of the distribution. This is consistent with its relatively strong $\mathrm{H}_{\alpha}$ emission, which in fact places it on the borderline with CTTSs according to the classification scheme of Briceño et al. (2005).

The same field was again observed in the same way over seven clear nights between 2010 December 8 and 17. The transit events 
were again evident with the same period and depth. Owing to improvements in the PTF image processing software, better weather, and mitigation of the CCD fogging effect seen in the previous year's data (see van Eyken et al. 2011; Ofek et al. 2012), the rms noise floor for the brightest stars in the 2010 December data set improved from $\approx 4$ to $\approx 3 \mathrm{mmag}$, with less evidence of systematic effects. ${ }^{19}$ Figures 2 and 3 show the light curves obtained in the first and second years of observations with PTF. The quasi-periodic stellar variability is evident, along with sporadic low-level flaring, consistent with the star's young age and late spectral type. Visual inspection of the curves revealed additional regular periodic transit-like signature, with a depth of $\approx 3 \%-4 \%$, superposed on the stellar variability. We initially determined an approximate period $(\approx 0.45$ days $)$ and transit duration $(\approx 2 \mathrm{hr})$ by hand, in order to locate all the transit windows. A detailed discussion of the light curves and derived properties is given in Section 3.1. The regularity and planet-like characteristics of the transit events led us to obtain follow-up observations.

\subsection{Keck Adaptive Optic Imaging}

The PTF imaging instrument is seeing limited and has a pointspread function (PSF) with typical full width at half-maximum (FWHM) of $2^{\prime \prime} .0$, or $660 \mathrm{AU}$ at the estimated distance of the Orion OB1a and 25 Ori associations $(330 \mathrm{pc}$; Briceño et al. $2005,2007)$. We obtained adaptive optics (AO) images using the NIRC2 camera (PI: K. Matthews) on the $10 \mathrm{~m}$ Keck II telescope in order to probe regions in the immediate vicinity of the star and to search for any sources (false positives) that could mimic the signal of a transiting planet, such as a nearby eclipsing binary. At $R=15.2 \mathrm{mag}$, PTFO $8-8695$ is sufficiently bright for natural guide star observations and does not require the use of a laser for atmospheric compensation. We locked the AO system control loops onto the target using a frame rate of $41 \mathrm{~Hz}$. The air mass was 1.29. We used the NIRC2 narrow camera setting to provide fine spatial sampling $\left(10\right.$ mas pixel $\left.^{-1}\right)$. Our observations consisted of 12 dithered $H$-band images ( 3 coadds per frame, $10 \mathrm{~s}$ per co-add), totaling 6 minutes of on-source integration time. Raw frames were processed by cleaning hot pixels, subtracting background noise from the sky and detector, and aligning and co-adding the results.

Figure 4 shows the final reduced image using an effective field of view of $4^{\prime \prime} 9 \times 4$.'.9, which corresponds to $\approx 4.9$ PTF pixels on a side. The image shows no contaminants, except for one faint source to the southeast, at a separation of 1".8 (590 AU in the plane of the sky at the distance of Orion OB1a/25 Ori). Our diffraction-limited images (FWHM $\approx 80$ mas) rule out additional off-axis sources down to a level of $\Delta H=4.3,6.4,8.9$, and $9.1 \mathrm{mag}(3 \sigma)$ at angular separations of $0^{\prime \prime} .25,0^{\prime \prime} .5,1^{\prime \prime} .0$, and $2^{\prime \prime} .0(83,165,330$, and $660 \mathrm{pc})$, respectively. Assuming a color difference of $0 \mathrm{mag}$, a faint, blended, $100 \%$ eclipsing binary would have to be within $\Delta H \approx 3.5-3.8$ mag of the primary star, or brighter, to mimic the observed transit depth at $R$ of $\approx 3 \%-4 \%$. At 6.96 mag (608 times) fainter than our target, the one-imaged contaminant is too faint to be a blended eclipsing binary capable of mimicking the observed transits unless it is extremely blue $(R-H \lesssim-3.2)$; in that event, however, it would be unlikely to be stellar in origin in any case. Any other such binary would have to lie within 0.25 of our target in order not to have been detected.

\footnotetext{
19 Independent differential photometric analyses of other PTF data, and respective precision levels, are discussed in Agüeros et al. (2011) and Levitan et al. (2011).
}

\subsection{LCOGT, KPNO $4 \mathrm{~m}$, and Palomar 200" Vetting}

Photometric observations were obtained in 2011 February with the Las Cumbres Observatory Global Telescope Network (LCOGT), using the $2 \mathrm{~m}$ Faulkes North and South telescopes, and the $0.8 \mathrm{~m}$ Byrne Observatory telescope at Sedgwick Reserve (BOS). We were able to confirm complete transit detections on two separate nights with the BOS telescope with a clear filter (see Figure 5). Observations in SDSS $g^{\prime}$ and $i^{\prime}$ with the three telescopes were inconclusive, and largely hampered by poor observing conditions and sparse phase coverage. The LCOGT observations enabled us to confirm the transit event and its period independently of the PTF data, and, in combination with the PTF data, we were able to establish a more accurate and up-to-date transit ephemeris.

In addition we obtained low-precision follow-up RV observations with the KPNO $4 \mathrm{~m}$ Mayall telescope and the Palomar $200^{\prime \prime}$ Hale telescope over 3 and 2 consecutive nights, respectively, to allow us to rule out a stellar-mass companion as the cause of the transits. The separate confirmation of the transit event and the lack of RV signature at the level of $\sim 10 \mathrm{~km} \mathrm{~s}^{-1}$ suggested that we had indeed detected a sub-stellar object, and we pursued additional observations.

\subsection{HET and Keck Spectroscopy}

Following the AO vetting and low-precision RV followup, we obtained Doppler RV spectroscopy with both the High Resolution Spectrograph (HRS; Tull 1998) on the $9.2 \mathrm{~m}$ Hobby-Eberly Telescope (HET; Ramsey et al. 1998), and the High Resolution Echelle Spectrometer (HIRES; Vogt et al. 1994) on the $10 \mathrm{~m}$ Keck I telescope. The goal was to detect or place an upper limit on any signal due to reflex stellar motion caused by the orbiting companion.

The queue-schedule operation mode of the HET (Shetrone et al. 2007) allowed us to obtain four spectroscopic RV observations very quickly after analysis of the low-precision RV and LCOGT photometric vetting data. The observations were timed on the basis of prior knowledge of the transit ephemeris to best constrain any orbital signature.

Observations from the fiber-fed HRS spectrograph and calibrated using ThAr exposures provide RV precision of better than $50 \mathrm{~m} \mathrm{~s}^{-1}$ over timescales of several weeks (Bender et al. 2012). Seeing-induced PSF changes are minimal due to the image scrambling properties of optical fibers (Heacox 1987) and the HRS temperature is kept stable to $\sim 0.01^{\circ} \mathrm{C}$. We used the $15 \mathrm{k}$ resolution mode of HRS, with the red cross-disperser setting (316g7940), giving a wavelength coverage of 6114-9861 A. The use of this lower resolution setting enhances the efficiency of HRS without significantly degrading the information content in the stellar spectrum (since the target absorption lines were already known to be rotationally broadened). Each observation was $1200 \mathrm{~s}$ in duration, except on UT 2011-02-21, which was $1600 \mathrm{~s}$, and ThAr calibration frames were taken immediately after each observation to track instrument drift. Data for the four nights were reduced in IDL with a custom optimal extraction pipeline that performs bias subtraction, flat fielding, order tracing and extraction, and wavelength calibration of the extracted one-dimensional spectra. A simultaneous sky-fiber was available with this spectrograph setting, but we chose to avoid the additional complexity inherent in performing sky subtraction with a fiber-fed spectrograph, and instead masked areas affected by skylines so that they were not used in calculating the RVs. The achieved signal to noise in each spectrum was 

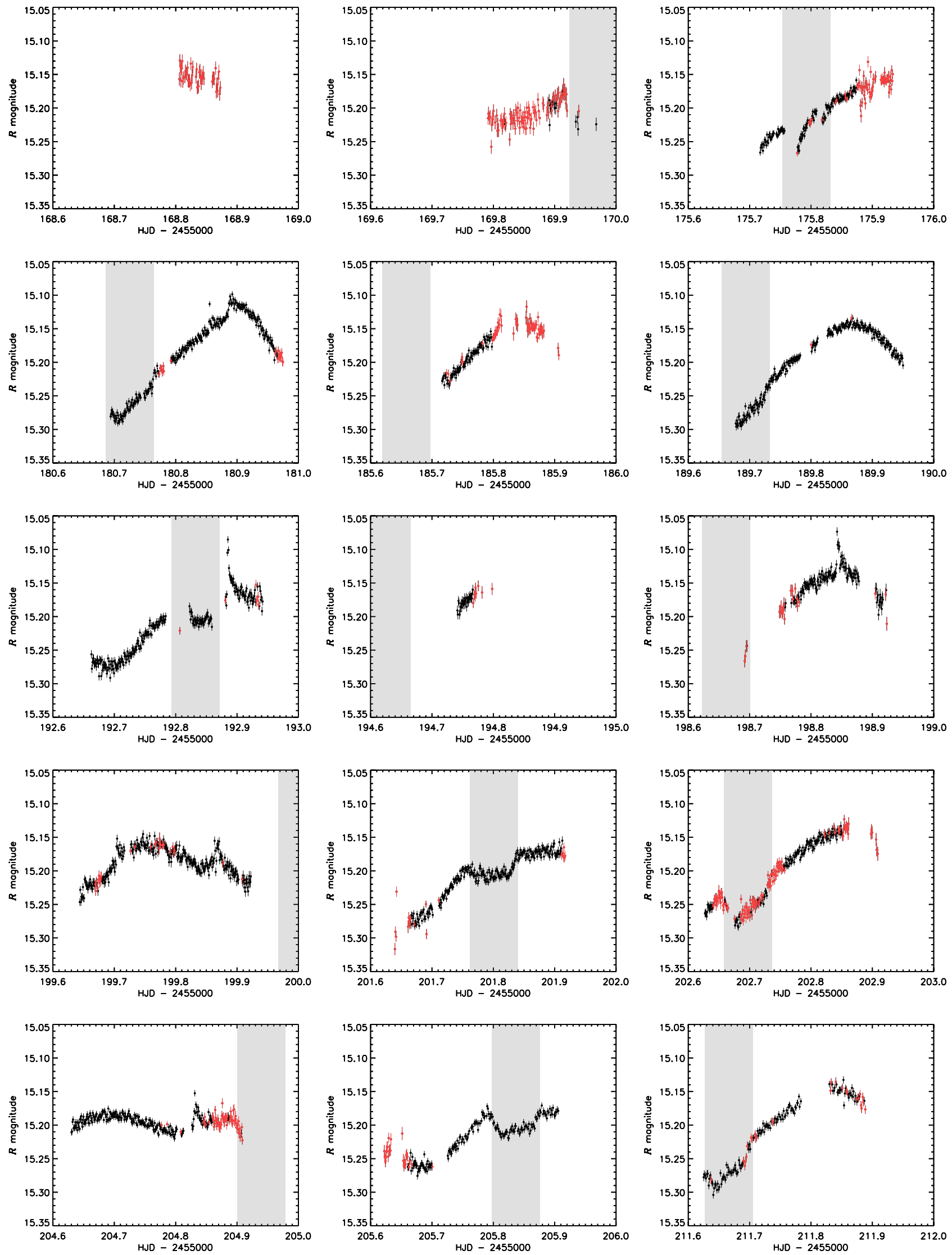

Figure 2. Photometric light curve for 2009 December 1-2010 January 15 (Sections 2.1 and 3.1.1). Light gray regions indicate the transit windows, fixed at the measured transit period, width, and epoch of center-transit $\left(T_{0}\right)$. Dark gray points (red in the online journal) indicate data automatically flagged by the data reduction software as potentially non-optimal for various possible reasons (e.g., imperfect weather, evidence of contamination within the photometric aperture, etc.).

(A color version of this figure is available in the online journal.) 

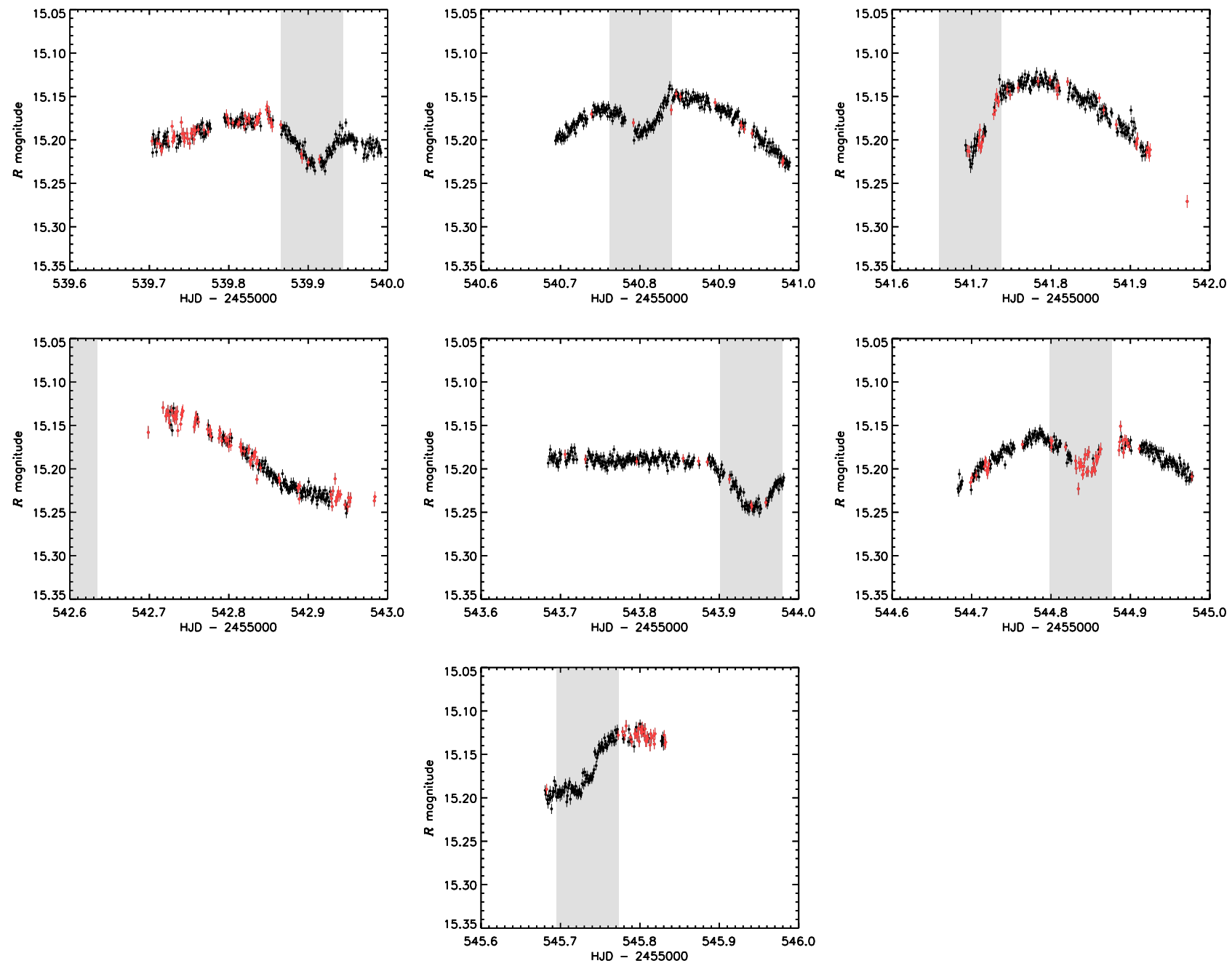

Figure 3. Same as Figure 2, for 2010 December 8-17.

(A color version of this figure is available in the online journal.)
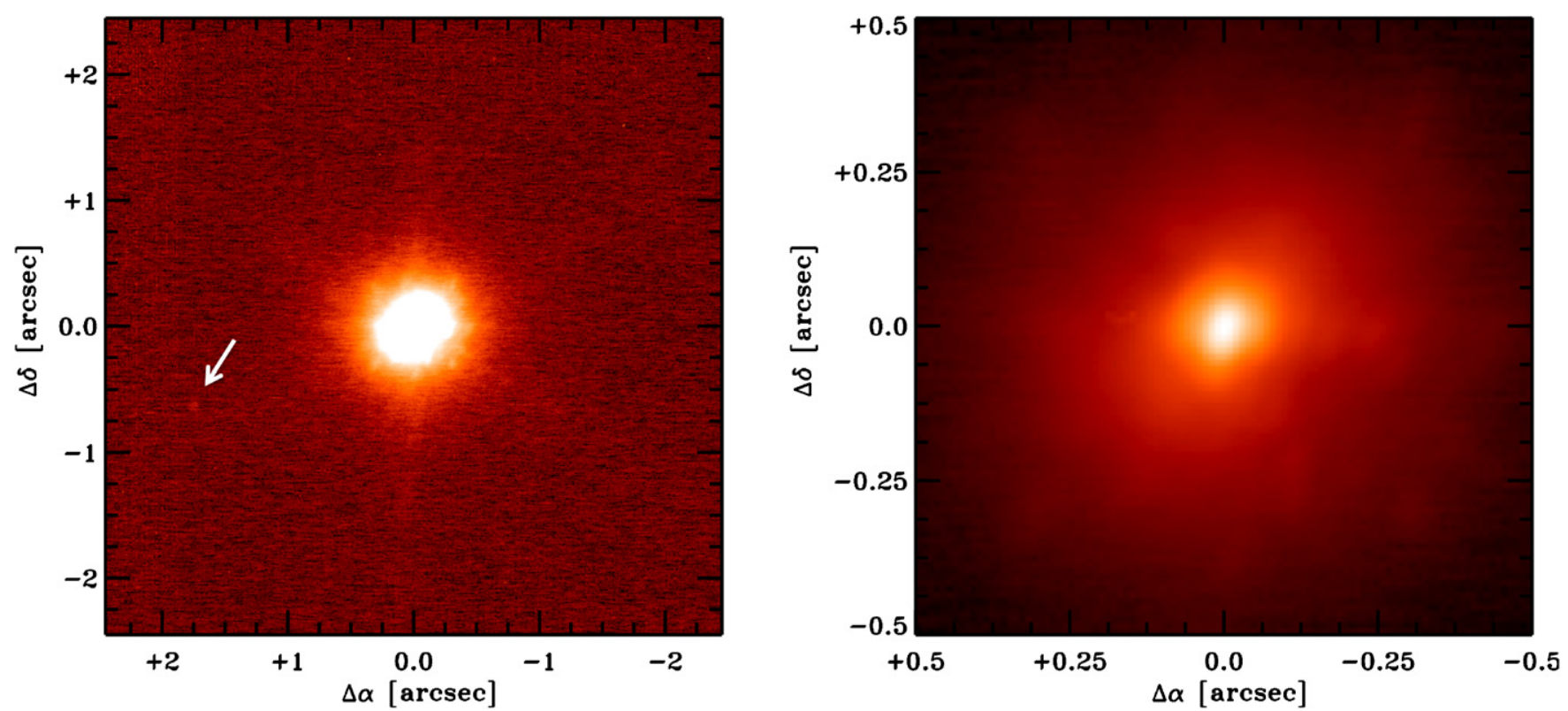

Figure 4. Adaptive optics imaging of PTFO 8-8695, showing the full image field (left), and detail around the central star (right), at two different logarithmic brightness stretches. A very faint source is detected to the south east of the central star at a separation of 1".8, 6.96 mag fainter than the central source, and unlikely to be capable of mimicking the observed transits (indicated by the arrow, left panel); otherwise no contaminants are detected. (Section 2.2.).

(A color version of this figure is available in the online journal.) 

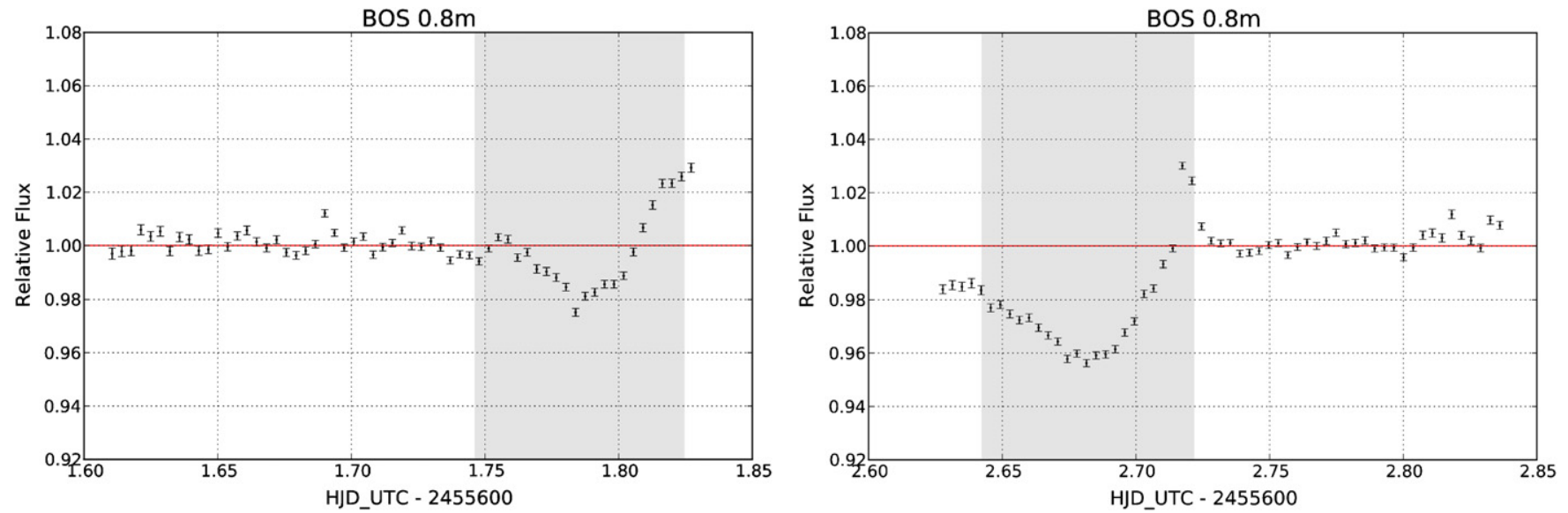

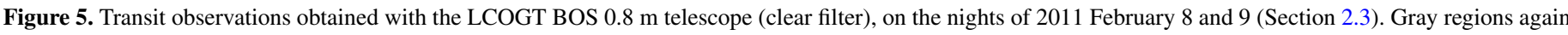

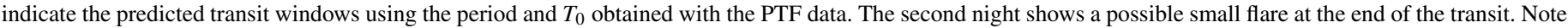
that in addition to stellar variability, some of the systematic trends may also be air-mass related.

(A color version of this figure is available in the online journal.)

approximately 40-50 per resolution element, peaking in the $I$ band. We identified the strongest absorption lines in an M2-type high resolution Kurucz stellar model ${ }^{20}$ (Kurucz 2005), and used these to select lines for fitting in the measured spectra.

For the Keck data, we observed PTFO 8-8695 using the standard procedures (e.g., Howard et al. 2009) of the California Planet Search for HIRES. The five observations over 10 days were each 500-600 s in duration and achieved a signal-to-noise ratio of $10-20$ per pixel ( $\mathrm{S} / \mathrm{N}=20-40$ per resolution element) in the $V$ band. We used the "C2" decker ( 0 ".86 wide slit) for a spectral resolution of $\sim 60,000$. The total wavelength coverage was $\approx 3650-7970 \AA$.

Owing to the faintness of the target and the relatively relaxed precision requirements, we employed in both cases thorium-argon (ThAr) emission lamps as the fiducial reference. This avoids the light-throughput penalty incurred by the higher precision common-path iodine gas absorption cell, which is better suited to brighter targets.

Data reduction and analysis were performed independently at separate institutions for the two data sets, using independently written software, but following the same general principles. Visual inspection of the spectra readily revealed strong rotational line broadening $\left(v_{\mathrm{r}} \sin i_{*}=80.6 \pm 8.1 \mathrm{~km} \mathrm{~s}^{-1}\right.$-see Section 3.2 .2 ), to the extent that only the very strongest of the photospheric lines were evident above the noise level. The low signal-to-noise ratio $(\mathrm{S} / \mathrm{N})$ and high stellar rotational velocity resulted in a very small number of lines from which to measure the RV. Using a cross-correlation analysis on the HRS spectra to measure these broad, low $\mathrm{S} / \mathrm{N}$ lines yielded a velocity uncertainty of $\sim 10-14 \mathrm{~km} \mathrm{~s}^{-1}$. Such an analysis is very sensitive to bad pixels or faint skylines, which were difficult to remove in the low $\mathrm{S} / \mathrm{N}$ regime. In addition, a cross correlation strongly benefits by combining the information content of multiple lines with fixed relative spacings. In our data, viable lines tended to be spaced far apart: Typically there were only one or two lines per spectral order, which negated much of the advantage of using a cross-correlation. Consequently, we adopted an alternative approach of fitting individual stellar lines with Gaussian functions, solving each line independently for its center, width, and depth. This fitting procedure was much less sensitive than crosscorrelation to bad pixels or to the precise spectral window being

\footnotetext{
20 http://kurucz.harvard.edu
}

Table 2

Differential Radial Velocity Measurements

\begin{tabular}{lrc}
\hline \hline HJD & \multicolumn{1}{c}{$\begin{array}{c}\mathrm{RV} \\
\left(\mathrm{km} \mathrm{s}^{-1}\right)\end{array}$} & Telescope \\
\hline 2455613.668022 & $1.81 \pm 0.64$ & HET \\
2455615.649694 & $2.41 \pm 0.64$ & HET \\
2455616.640274 & $-2.36 \pm 0.64$ & HET \\
2455623.622875 & $0.55 \pm 0.64$ & HET \\
2455663.744361 & $-1.53 \pm 0.91$ & Keck \\
2455670.747785 & $-0.01 \pm 0.96$ & Keck \\
2455671.755651 & $-1.38 \pm 1.03$ & Keck \\
2455672.757479 & $0.10 \pm 1.02$ & Keck \\
2455673.770857 & $2.83 \pm 1.33$ & Keck
\end{tabular}

Notes. RV uncertainties listed are formal uncertainties, and do not account for systematic effects. The offset between the two data sets is arbitrary, and set by shifting each set so that its mean is zero, excluding one outlier data point-see Sections 2.4 and 3.2.1

analyzed. It also allowed us to mask out telluric absorption and strong, narrow night sky emission lines.

The faintness of the target and the small semimajor axis of the putative companion's orbit meant that very high RV precision was neither anticipated nor required. Simple Gaussian+linearterm profiles provided an adequate fit (i.e., reduced $\chi^{2} \sim 1$ ) owing to the low $\mathrm{S} / \mathrm{N}$ and heavy broadening (and probable blending) of the lines, where more sophisticated models would have added unnecessary and unconstrained extra parameters. A weighted average of the shifts in the fit centroids with respect to the rest wavelengths of the stellar lines provided the required Doppler shifts. Since substantially differing $\mathrm{S} / \mathrm{N}$ in the various lines precluded a simple standard-deviationbased estimate of the measurement errors, errors were instead estimated by propagating the formal errors from the Gaussian fits to the lines. In the case of the Keck data, corrections were made for measured variation in the telluric lines to account for residual uncalibrated instrument drift, but these corrections were found to be negligible. A total of 14 absorption features spanning 5300-7700 $\AA$ were fit in the Keck data, and 14 spanning $\sim 6100-8700 \AA$ in the HET data. The measured RVs are listed in Table 2. 


\section{DISCUSSION}

\subsection{Photometry}

\subsubsection{Light Curves and Periodicities}

In order to model the transit events for a derivation of the transit and planetary candidate properties, the effects of the stellar variability in the light curves need to minimized-i.e., the light curves outside of transit needed to be whitened. After removing data points which are flagged or have large measurement errors, we fit a smooth cubic spline to all the PTF data which fall outside the transit windows (using the IDL imsl_cssmooth function $^{21}$ ), interpolating across the windows themselves. The fit is then subtracted (in magnitude space) from the entire light curve. Since most of the stellar variability occurs on longer timescales than the transits (with the exception of the occasional flares), this process yields the "whitened" light curve that has the majority of the stellar variability removed, leaving only the transits. Using a standalone version of the NASA Exoplanet Science Institute (NExScI) periodogram tool, ${ }^{22}$ we calculate the Plavchan periodogram (Plavchan et al. 2008) of the combined whitened data sets, to provide a more accurate formal transit period measurement. We find a clear peak at $0.448413 \pm 0.000040$ days. $^{23}$

Figure 6 shows the whitened data for the nights where any intransit data were obtained, folded on the measured period. Shortterm stellar variability is not corrected and is probably the most likely explanation for those transits (particularly those from JDs 2455175,2455192 , and 2455545) which deviate significantly in shape from the general form of the others. This may be caused by a combination of low-level flaring, residual disk occultation, and the companion transiting small-scale stellar surface features. JD 2455192 appears to show a flare immediately after the transit, and also likely the tail of a second flare mid-transit, though the gaps in the data prevent a clear interpretation. JD 2455545 appears to show an early transit egress; however, the mid-transit variation and the disparity in comparison to other transits in the same year are suggestive of the above-mentioned variability effects, which may confuse and mask the true egress time.

The original "un-whitened" light curve is dominated by stellar variability (see Figures 2 and 3). If we assume that the largescale variability is caused primarily by spot modulation, and that the relative effect of the transit events is negligible, we can use the unwhitened data to investigate the stellar rotation. Lomb-Scargle periodograms (Lomb 1976; Scargle 1982) of the two years' data are shown in Figure 7. Where the Plavchan periodogram is designed for finding regular periodic features of arbitrary but constant shape (such as a transit), a Lomb-Scargle periodogram is better suited for finding periodic modes in a more complex signal such as the quasi-periodic stellar variation in our data: Here, the signal appears not to repeat in an exact fashion and therefore does not fold well at any period (likely because of phase shifts due to changing spot features).

A strong peak is found at $0.4481 \pm 0.0022$ days, in fact matching well with the transit period. Another peak is seen at $0.9985 \pm 0.0061$ days, corresponding closely to the sidereal or

\footnotetext{
21 From the IDL Advanced Math \& Stats module; see http://www.ittvis.com/idl

22 See http://exoplanetarchive.ipac.caltech.edu

23 We make the assumption that there is no phase shift in the transit timing between the first and second years, such that the data sets can be combined to give a year-long time baseline, and thus a very precise period estimate. If we disregard this assumption, the first year's data alone give the best period estimate, $P=0.4486 \pm 0.0010$ days.
}

solar Earth day, and therefore most likely an artifact resulting from the observing cadence. The other peaks all appear to be aliases of these two periods. To confirm this, we modeled the data by creating an artificial light curve from two summed sinusoids with the same two periods, providing power at the frequencies of these two peaks. We superimposed artificial transits modeled as a simple inverted top-hat function, with the same depth, width, and ephemeris as the real transits. Allowing the phases and relative amplitude of the 0.4481 day and 0.9985 day signals to vary as free parameters, and requiring that the total amplitude of the light curve remains approximately the same as that of the actual data, we performed a least-squares fit to the real periodogram to assess how well its structure could be reproduced. Figure 7 shows the results, with a good match between model and data. Repeating the test with the 0.4481 day sinusoid omitted (i.e., summing only the one-day signal and the transits) gave a poor fit, implying that the form of the periodogram cannot be explained by the transits alone. Since there is clearly strong correlated out-of-transit variability associated with the star, and there are no other fundamental periods evident in the periodogram, the 0.4481 day signal appears to be the only likely period for the star.

There are three other notable aliases of the presumed stellar rotation period, $P_{*}$, at $0.3092 \pm 0.0010$ days, $0.8126 \pm 0.0070$ days, and $4.43 \pm 0.31$ days (identified in the figure). The larger of these is substantially above the upper period limit implied by the measured $v_{\mathrm{r}} \sin i_{*}$ of the star (Section 3.2.2), $P_{*}<2 \pi R_{*} /\left(v_{\mathrm{r}} \sin i_{*}\right)=0.671 \pm 0.092$ days. Though it is unlikely that the star would be coincidentally rotating at an alias of the transit period with Earth's rotation, we repeated the modeling experiment with the model stellar rotation modified to match the remaining two aliases to ensure that none could in fact be the true fundamental stellar period (see Dawson \& Fabrycky 2010). Similar periodograms were obtained, with peaks at similar locations but with differing strength ratios; none were as good a fit to the periodograms based on the real data. We therefore conclude that the star is corotating or near corotating with the companion orbit.

We note that it is difficult to model the observed transit events with star spots alone, particularly in the 2009 December/2010 January data: The short transit duration relative to the period, the flat bottom of the transits, and the sharp ingress and egress, are all more characteristic of a transiting object, whereas spots tend to cause smoother, more sinusoidal features as their projected area changes as they rotate across the stellar disk. It is more likely that we are seeing in the total light curve the effects of both star spots and a transiting object.

\subsubsection{Transit Fitting}

We fit transit models to the whitened light curves using the IDL-based Transit Analysis Package (TAP; Gazak et al. 2012). TAP employs Bayesian Markov Chain Monte Carlo (MCMC) techniques to explore the fitting parameter space based on the analytic transit light curve models of Mandel \& Agol (2002). The package incorporates white- and red-noise parameterization (Carter \& Winn 2009), allowing for robust estimates of parameter uncertainty distributions. We fit only the transits from nights where a complete transit was observed with adequate coverage both before and after the transit window, since on these nights the spline fit to remove the stellar variability is reasonably constrained on both sides of the transit; on nights where there is partial transit coverage, the spline fit tends to diverge during transit time. We also reject the transits mentioned 

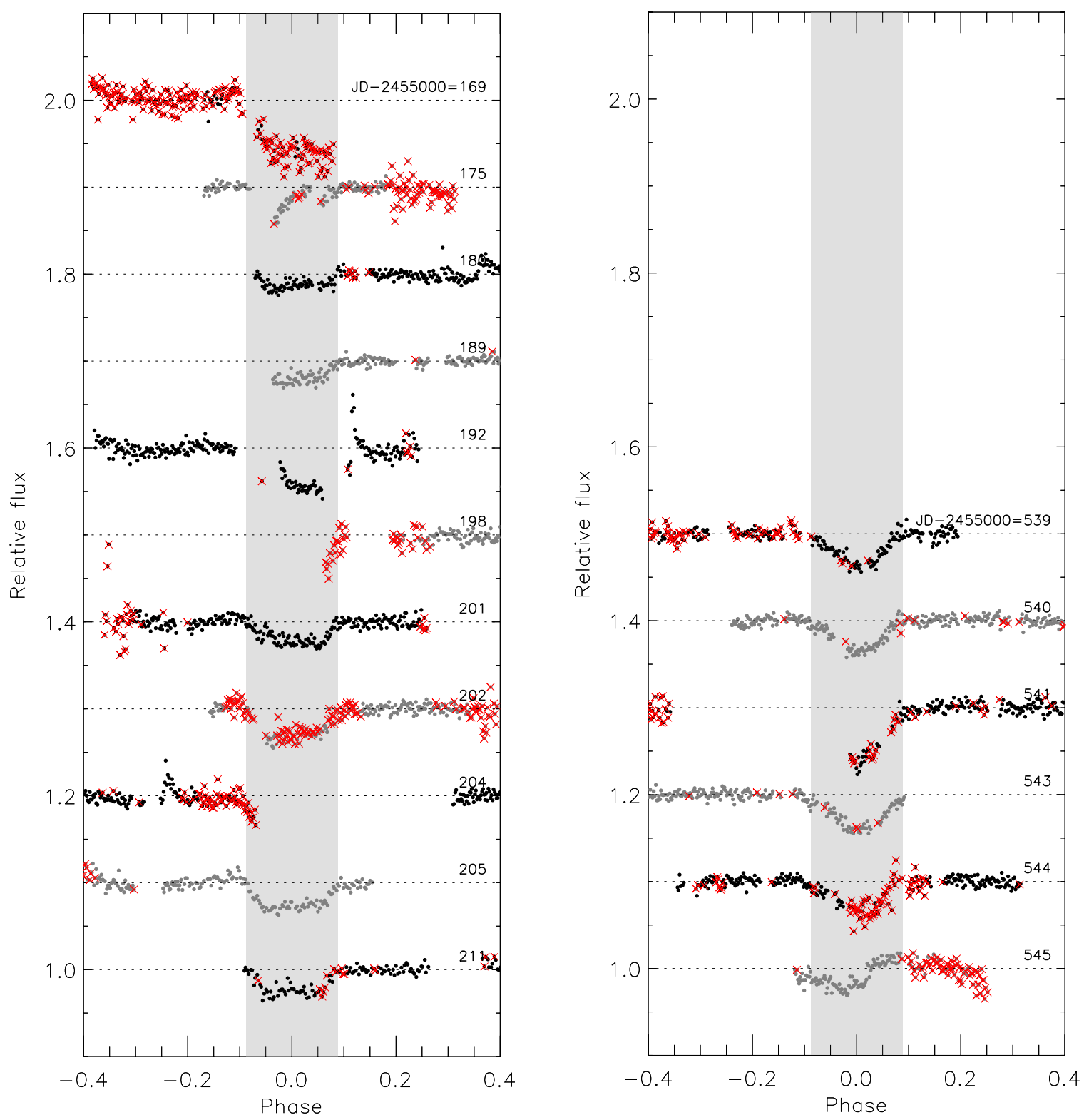

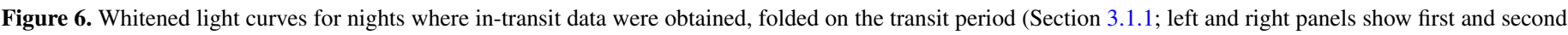

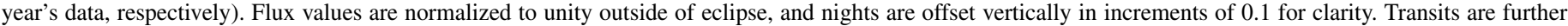

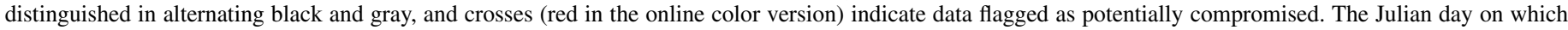

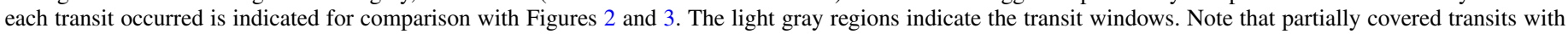
little or no data on one or other side of the transit window are likely to suffer from poor stellar-variability correction and show significant systematic error.

(A color version of this figure is available in the online journal.)

in Section 3.1 where stellar variability appears to strongly affect the shape of the transit, and in addition, the transit from JD 2455544, where the spline subtraction was particularly uncertain and rather sensitive to the tightness of the spline fit. Thus, we fit four of the transits from the first year's data (JDs 2455201, 2455202, 2455205, and 2455211), and three from the second year (JDs 2455539, 2455540, and 2455543). The folded data are shown in Figure 8.

For the transit fitting, we hold the orbital period, $P$, fixed to the transit period determined above. The eccentricity, $e$, is fixed at zero, since it is difficult to constrain in the absence of a secondary eclipse. White and red noise levels are allowed to float separately for each individual transit, as are linear air-mass trends. The remaining parameters are allowed to float, but are tied across all transits. They include: the epoch of transit center,
$T_{0}$ (we assume there are no transit timing drifts between the two years); the orbital inclination, $i_{\text {orb }} ; a / R_{*}$, where $a$ is the orbital semimajor axis, and $R_{*}$ is the radius of the primary; $R_{\mathrm{p}} / R_{*}$, where $R_{\mathrm{p}}$ is the companion radius; and the linear and quadratic limb-darkening coefficients for the primary star. In addition, we constrain the fitting such that $R_{\mathrm{p}} / R_{*}<1$, and white and red noise components are less than $4 \%$ (the depth of the transit). Parameters are calculated by fitting Gaussians to the dominant peak in the probability density distributions for each parameter resulting from the MCMC analysis. The center location of the Gaussian is taken as the parameter value, and measurement errors are estimated as the dispersion of the fit. We find that the limb-darkening coefficients are largely unconstrained by the data. The fit is shown overlaid on the folded data in Figure 8; the corresponding parameters are listed in Table 3. 


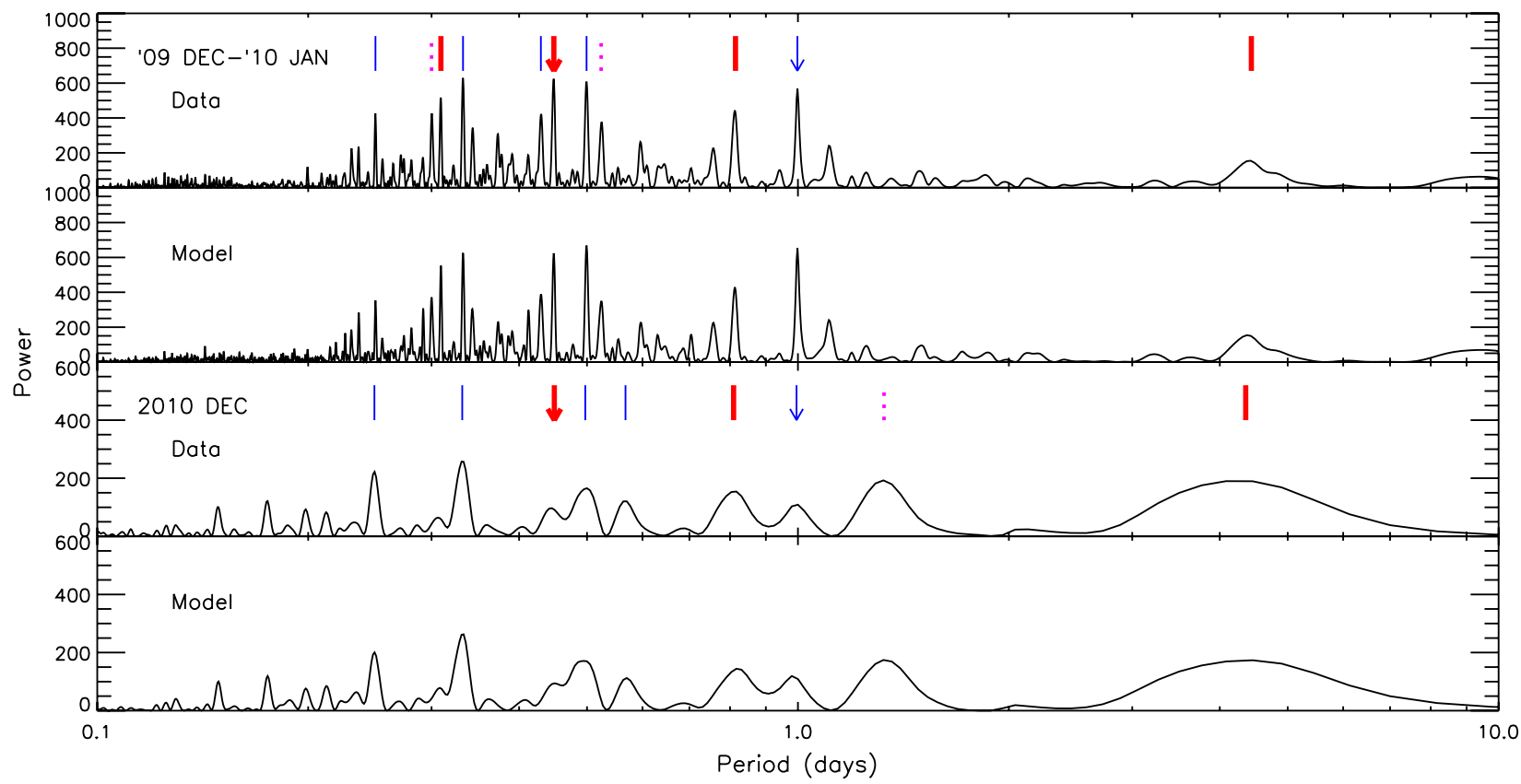

Figure 7. Lomb-Scargle periodogram of the non-whitened light curves from the two years of PTF Orion observations, compared to simple synthetic models (Section 3.1.1). The presumed stellar rotation period is marked with a thick arrow (red in the online color version); a second distinct one-day period, which we attribute to the observing cadence, is marked with a thin (blue) arrow. The more prominent aliases of these periods are indicated with vertical lines: thick (red) marks indicate peaks which are predominantly produced by aliases of the presumed stellar rotation period; thin (blue) marks indicate peaks predominantly produced by aliases of the one-day period. Dotted (magenta) lines indicate peaks produced by a combination of the two.

(A color version of this figure is available in the online journal.)

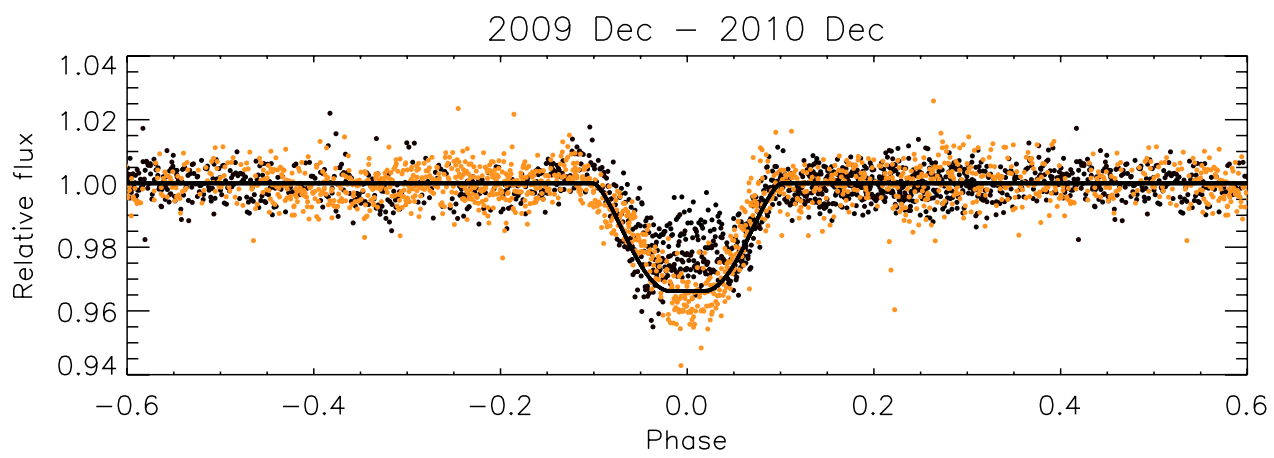

Figure 8. Folded light curve for the two years of observations combined, after removing stellar variability (Section 3.1.1). The first year's data (2009 December-2010 January) are plotted in black; the second year's (2010 December) are plotted in gray (orange in the online journal). A change in transit shape between the two years is evident. The best transit fit to both years combined is overplotted on the assumption that $R_{p}<R_{*}$ (Section 3.1.2). Limb darkening is neglected, and error bars are omitted for clarity; the median photometric error is 0.0046 , with an out-of-eclipse standard deviation of 0.0056 .

(A color version of this figure is available in the online journal.)

There is significant variation in the light curve from transit to transit, as can be seen in Figure 6. This can reasonably be attributed to the companion passing across varying surface features - cold or hot spots, or perhaps flares—on the stellar photosphere. Such variations are a result of the planet tracing the stellar surface brightness profile as it traverses the stellar disk, and cannot be removed by the whitening process, which is only sensitive to the integrated brightness of the disk. Since PTFO 8-8695 is expected to be active and spotted, such variation in the transits is further suggestive of a genuine transit, rather than a background blend.

It can also clearly be seen from Figures 6 and 8, however, that there is an overall change in the transit shape between the two years' data sets. Explanations for this remain speculative. Re-running the fitting process and allowing a change in both $R_{*}$ and $R_{\mathrm{p}}$ between the two years yields a decrease in stellar radius of $\approx 10 \%(2.1 \sigma)$ from one year to the next (with no measurable change in companion radius). Such a large change in the stellar radius in such a short time period is unlikely, and would likely manifest itself in observable rotation rate changes not seen in the data. A change in transit shape could also arise in principle from a change in orbital geometry. For example, the host star is rotating quickly enough to exhibit significant oblateness, which may induce precession of the orbital plane and, therefore, changes in $i_{\text {orb }}$ if the orbital and stellar rotational axes are misaligned. ${ }^{24}$ An additional planet in a different orbit could also produce a similar effect (Miralda-Escudé 2002).

\footnotetext{
${ }^{24}$ Given the small orbital period, this may occur on short timescales. Following Miralda-Escudé (2002), we estimate the order of magnitude of the oblateness-induced gravitational quadrupole moment of the host star, $J_{2}$, by scaling to the pre-main sequence from a solar value of $\sim 10^{-7}$ to $10^{-6}$ according to $J_{2} \propto R_{*}^{3} /\left(M_{*} P_{*}^{2}\right)$. Assuming that the stellar obliquity is small, and noting that the orbital angular momentum and stellar rotational angular momentum are similar for $M_{\mathrm{p}} \sim 5 M_{\mathrm{Jup}}$ (so that the inclinations of the stellar rotation and orbital planes with respect to the mean plane are similar), this leads to a precession period of the orbital nodes on the order of tens to hundreds of days.
} 
Table 3

System Parameters

\begin{tabular}{lc}
\hline \hline Parameter & \multicolumn{1}{c}{ Value } \\
\hline$P$ & Measured \\
$i_{\text {orb }}$ & $0.448413 \pm 0.000040$ days \\
$a / R_{*}$ & $1.685 \pm 0.064$ \\
$R_{\mathrm{p}} / R_{*}$ & $0.1838 \pm 0.0097$ \\
$T_{0}(\mathrm{HJD})$ & $2455543.9402 \pm 0.0008$ \\
$v_{\mathrm{r}} \sin i_{*}$ & $80.6 \pm 8.1 \mathrm{~km} \mathrm{~s}^{-1}$ \\
\hline & Derived \\
\hline$a$ & $0.00838 \pm 0.00072 \mathrm{AU}$ \\
$R_{*}$ & $=1.80 \pm 0.15 R_{\odot}{ }^{\mathrm{a}}$ \\
$R_{\mathrm{p}}$ & $1.07 \pm 0.10 R_{\odot}$ \\
$M_{\mathrm{p}} \sin i_{\text {orb }}$ & $1.91 \pm 0.21 R_{\mathrm{Jup}}$ \\
$M_{\mathrm{p}}$ & $\leqslant 4.8 \pm 1.2 M_{\mathrm{Jup}}$ \\
\hline
\end{tabular}

Notes. Summary of parameters determined in this paper for the PTFO 8-8695 system. Quantities are $P$ : orbital period; $i_{\text {orb }}$ : orbital inclination; $i_{*}$ : inclination of stellar rotation axis; $a$ : orbital semimajor axis; $R_{*}$ : stellar radius; $R_{p}$ : planet radius; $T_{0}$ : epoch of transit center; $v_{\mathrm{r}}$ : stellar equatorial rotational velocity; $M_{\mathrm{p}}$ : planet mass.

${ }^{\text {a }}$ From Kepler's third law, assuming stellar mass $M_{*}=$ $0.39 \pm 0.10 M_{\odot}$ (Briceño et al. 2005), and $M_{\mathrm{p}} \ll M_{*}$

${ }^{\mathrm{b}}$ Upper limit derived from measured RV semi-amplitude.

It is difficult, however, to explain how an inclination change can yield a transit that becomes both more grazing (longer ingress/egress) and deeper at the same time. Another explanation may be variation in star spot coverage (or limb-darkening, though with heavy spot coverage, the two effects become somewhat confused). In addition to short-term spot variations, there may be surface features which survive for much longer periods (see, e.g., Mahmud et al. 2011). Given the activity of the star and the extreme proximity of the companion, such features may be compounded by magnetic or tidal star/planet interactions that could give rise to varying hot or cold spots near the subplanetary point on the stellar photosphere. This could affect the apparent shape of the transit in a systematic way. The planet's apparent proximity to the tidal disruption limit (see Section 3.3) may also be a factor: A tidally distorted shape, or transient rings or a tidal tail of evaporating material could all yield unexpected and possibly varying transit shape (and also cause the slight transit asymmetry seen in the second year's data). For the sake of simplicity, and given the lack of data to disentangle all of these possibilities, we here adopt the single combined fit to both years' data sets, although with the caveat that the variability may cause some systematic error in the measurements.

We note that our fit yields a somewhat smaller stellar radius, $R_{*} \approx 1.07 R_{\odot}$, than that previously reported by Briceño et al. (2005) $\left(1.39 R_{\odot}\right)$. Assuming that their estimate of $T_{\text {eff }}=$ $3470 \mathrm{~K}$ is correct, interpolating Siess models (Siess et al. 2000) with this updated radius gives a slightly older age estimate for the star, $\approx 3.7 \mathrm{Myr}$ versus $2.7 \mathrm{Myr}$. Given the distance uncertainties in the Briceño et al. (2005) results, however, and the possibility of uncertainty in $T_{\text {eff }}$ arising from heavy spotting in the stellar photosphere, the two radius estimates are probably not inconsistent. ${ }^{25}$

25 Our radius estimate also depends on our estimate of $a$, which depends in turn on the assumption that the mass estimate of Briceño et al. (2005) is correct, so the argument is somewhat circular. $a$, however, is relatively insensitive to

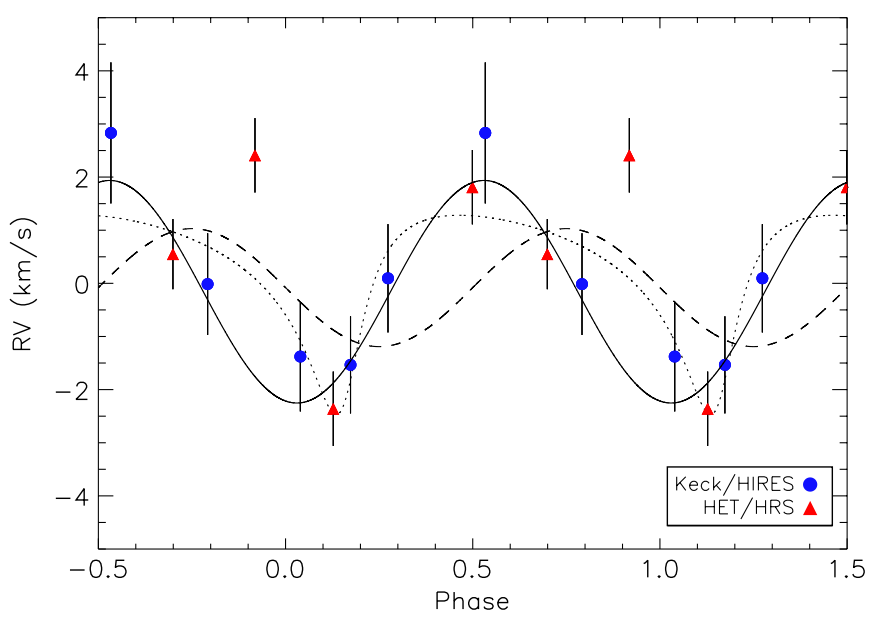

Figure 9. Differential radial velocity measurements obtained with Keck/HIRES and HET/HRS (Section 3.2.1). Zero phase is chosen to correspond to the photometric center-of-transit time. The offset between the two data sets is chosen to match the mean RV of each. The lines indicate best Keplerian fits (excluding the outlier): dashed line-circular orbit, transit-center time, $T_{0}$, fixed to photometry $\left(\chi_{\text {red }}^{2}=4.0\right)$; dotted line-eccentric orbit, transit-center time fixed to photometry $\left(\chi_{\text {red }}^{2}=1.1\right.$ ); solid line - a sinusoidal fit (equivalent to a circular orbit) at the same period, with phase free to float $\left(\chi_{\text {red }}^{2}=0.42\right)$. The eccentric fit is better than the fixed- $T_{0}$ fit, but brings the companion to the surface of the star at periastron. The floating-phase sinusoidal fit gives the best result, suggesting that star spots either modify or dominate the Doppler RV signal. The outlier point may represent Rossiter-McLaughlin effect due to the transiting companion.

(A color version of this figure is available in the online journal.)

\subsection{Spectroscopy}

\subsubsection{Radial Velocities}

Since the RV analysis is differential in nature, the RV offset between the HET and Keck data sets is arbitrary. We place them on the same approximate scale by shifting the RV zero points to the mean of the data for each data set. There are too few data points to create a periodogram to measure any periodicities in the data, but we can look for consistency with the previously determined transit period by folding the data on that period and looking for a coherent alignment of the data points. The result is shown in Figure 9: Indeed, the data appear to phase well, showing a smooth and apparently sinusoidal variation, with the exception of one outlying data point at orbital phase $\phi=-0.082$ (Heliocentric Julian date (HJD) 2455615.64969), which is discussed further below. This outlier is excluded in calculating the offset between the data sets.

To constrain the mass of the companion, we fit a Keplerian orbit model to the data using the RVLIN package by Wright \& Howard (2009). The model includes six parameters: the period, $P$; the RV semi-amplitude, $K$; the eccentricity, $e$; the argument of periastron, $\omega$; the time of periastron passage, $T_{\mathrm{p}}$; and the systemic velocity, $\gamma$. We fixed $P$ and $T_{\mathrm{p}}$ to the values measured from the transit photometry. Since the $\chi^{2}$ surface has multiple minima, we explore the fitting parameter space by running 10,000 trial Keplerian fits (neglecting the apparent outlier point), with initial parameter estimates drawn at random from reasonable starting distributions, and selecting the fit with the lowest reduced $\chi^{2}\left(\chi_{\text {red }}^{2}\right)$.

stellar mass $\left(a \propto M^{1 / 3}\right)$, and the Siess models predict a negligible difference in mass at our older age. (In fact, at the estimated $T_{\text {eff }}$, they predict a mass range smaller than the errors over an age range of $1-10$ Myr.) 
We find that the constraints from the transit ephemeris make it difficult to obtain a reasonable Keplerian fit. A good circular-orbit fit, with the eccentricity, $e$, fixed to zero, is prohibited by the constraint on $T_{0}$ provided by the photometry. Such a fit must cross its central velocity at phases 0 and 0.5 , with minima and maxima at 0.25 and 0.75 (the quadrature points), and as a result appears significantly out of phase with the data $\left(\chi_{\text {red }}^{2}=4.0\right.$; dashed line, Figure 9).

An eccentric fit goes some way to resolving the mismatch $\left(\chi_{\text {red }}^{2}=1.1\right)$, but yields an eccentricity of $e \approx 0.492$ (dotted line, Figure 9). This places the fractional periastron distance at $r_{\text {peri }} / a \equiv 1-e \approx 0.51$, bringing the companion to the point of contact with the surface of the star as determined from the photometric transit fits $\left(R_{*} / a \approx 0.51\right)$. Such a solution may be improbable, given that in Section 3.3 we note that the orbital semimajor axis appears to be close to the Roche limit.

A better fit is in fact obtained by fitting a circular orbit model and removing the constraint on $T_{0}$, allowing the phase to float. This fit is overplotted in Figure 9, showing the fit is good $\left(\chi_{\text {red }}^{2}=0.42\right)$, but offset in phase from the transit ephemeris by $-0.22 \pm 0.04$ periods.

We favor this better fit to the RV signal, which most likely arises because of spot effects modulated by the stellar rotation, where the amplitude of the spot effect is at least comparable to-if not much greater than-any true reflex Doppler signal from the companion. Similar spot-induced RV amplitudes have already been observed for other TTSs in the optical (e.g., Mahmud et al. 2011; Huerta et al. 2008; Prato et al. 2008). Since the star appears to be corotating with the planet orbit, the period of such a spot-dominated RV signal would match the orbital period, but the phase would be arbitrary, depending on the longitudinal spot placement. Alternatively, spot and companion RV signals may both be significant and the two effects may compete (see Section 3.3).

Regardless of the astrophysical cause behind the measured RV signal, we can place an upper limit on the companion mass, and because of the good orbital phase coverage we can be reasonably assured that possible aliasing effects are not a concern. We assume that any companion-induced Doppler motion must be smaller than the amplitude of the measured variations, that $M_{p} \ll M_{*}$, and that $M_{*}=0.39 \pm 0.10 M_{\odot}$ (Briceño et al. 2005), and hence estimate $M_{\mathrm{p}} \sin i_{\text {orb }} \leqslant 4.8 \pm$ 1.2 $M_{\text {Jup }}$ (see, e.g., formula 3 , Gaudi \& Winn 2007). Taking our derived value of $i_{\text {orb }}$ we can directly estimate $M_{\mathrm{p}} \leqslant 5.5 \pm$ $1.4 M_{\text {Jup }}$, comfortably within the planetary mass regime. The semi-amplitude (half peak-to-peak) of the combined measured $\mathrm{RV}$ variations is $\approx 2.4 \mathrm{~km} \mathrm{~s}^{-1}$. This is separately confirmed by the two individual data sets, which both have good phase coverage and show similar amplitudes independent of the RV offset (as can be seen in Figure 9). By comparison, since Doppler-induced RV semi-amplitude scales directly with $M_{\mathrm{p}}$, a $25 M_{\text {Jup }}$ object on the planet/brown dwarf boundary suggested by Schneider et al. (2011) would induce an $11 \mathrm{~km} \mathrm{~s}^{-1}$ signal; an object on the deuterium-burning limit $\left(\approx 13 M_{\text {Jup }}\right)$ would induce a $5.7 \mathrm{~km} \mathrm{~s}^{-1}$ signal. It is unlikely, therefore, that the object is of more than planetary mass, and still less in the stellar mass range. ${ }^{26}$ Since the spot distribution is likely to change with time, further observations to look for changes in the phase of the RV signal with respect to the transit ephemeris may provide more

\footnotetext{
26 We do note, however, that there is a possibility that if the reflex Doppler signature and the spot signature are of comparable amplitudes, matching periods, and opposed in phase, they may destructively interfere, giving an artificially low RV amplitude.
}

insight into the exact nature of the RV signal. RV observations in the infrared are also likely to suffer less from spot-induced noise, and so could also provide valuable further information.

We were unable to find any abnormalities in the observations regarding the outlying RV data point, and, thus, have no reason to reject it as a bad measurement. However, falling at an orbital phase $\phi=-0.082$, it lies within the transit window (cf., Figure 8), and its anomalous velocity could reasonably be explained as arising from the Rossiter-McLaughlin (RM) effect (Rossiter 1924; McLaughlin 1924). The RM effect would appear regardless of whether the main RV signal is caused by spot modulation or stellar reflex motion, since it is a result of an asymmetric distortion of the stellar absorption lines as an obscuration transits the unresolved stellar disk, blocking off regions with successively different rotational redshifts. Indeed, given the rapid stellar rotation, the RM effect should be expected to appear during the transit window. From Equation (6) of Gaudi \& Winn (2007) we can estimate the expected maximum amplitude of the effect, $K_{R}$, given $v_{\mathrm{r}} \sin i_{*}, R_{p}$, and $R_{*}$. We find $K_{R} \approx 3 \mathrm{~km} \mathrm{~s}^{-1}$, in good agreement with the offset of the outlier. The RM maximum also lies typically around $\sim 1 / 5-1 / 3$ of the way between transit-ingress and transit-center for gasgiant planets, which is coincidentally where the outlier point is located. The sign of the offset of the outlier data point, falling prior to the transit center time, is in agreement with a prograde orbit, as we would expect if the star is in synchronous (or quasi-synchronous) rotation. It should be noted that the RV data point at $\phi=+0.039$ (HJD 2455671.755651) also lies within the transit window but, in contrast, does not appear to be an outlier. Lying closer to the transit center time, one would expect the RV offset to be smaller here; however, the exact form of the RM effect depends on the precise orbital geometry, and may be partially masked by the choice of offset between the two data sets. Further complication may also arise from the companion transiting photospheric surface features. Dedicated RV measurements during transit could provide valuable confirmation of the RM effect hinted at by the data, and help independently confirm the validity of the transiting planet candidate.

\subsubsection{Stellar Rotation}

The stellar rotation velocity, $v_{\mathrm{r}}$, measured from the spectroscopy provides an independent consistency check on the stellar rotation period. In order to estimate the projected rotation velocity, $v_{\mathrm{r}} \sin i_{*}$, we degrade the Kurucz synthetic model spectrum (see Section 2.4) to the resolution and sampling of the HRS, and rotationally broaden it using a nonlinear limbdarkening model (Claret 2000; Gray 1992) over the range of $v_{\mathrm{r}} \sin i_{*}=10-150 \mathrm{~km} \mathrm{~s}^{-1}$. We then Doppler shift the broadened models, line by line, by cross-correlating against lines in our target spectrum. Subtracting a Doppler shifted model from the observed spectrum gives an rms residual for that rotational velocity. Minimizing this residual gives the optimal rotational velocity measured for an individual line. We applied this analysis to 12 lines that were sufficiently deep to be visible above the noise for our full range of $v_{\mathrm{r}} \sin i_{*}$ models. Weak lines could not be used because at large $v_{\mathrm{r}} \sin i_{*}$ the line profiles became buried in the noise. The weighted average of $v_{\mathrm{r}} \sin i_{*}$ measured for these lines, across all four HRS observations, was $80.6 \pm 8.1 \mathrm{~km} \mathrm{~s}^{-1}$.

The assumed rotational period of the star and the radius measured from photometric transit fitting (neglecting oblateness effects) independently imply an expected value for the unprojected rotational velocity of $v_{\mathrm{r}}=2 \pi R_{*} / P_{*} \approx 120 \pm 11 \mathrm{~km} \mathrm{~s}^{-1}$. 


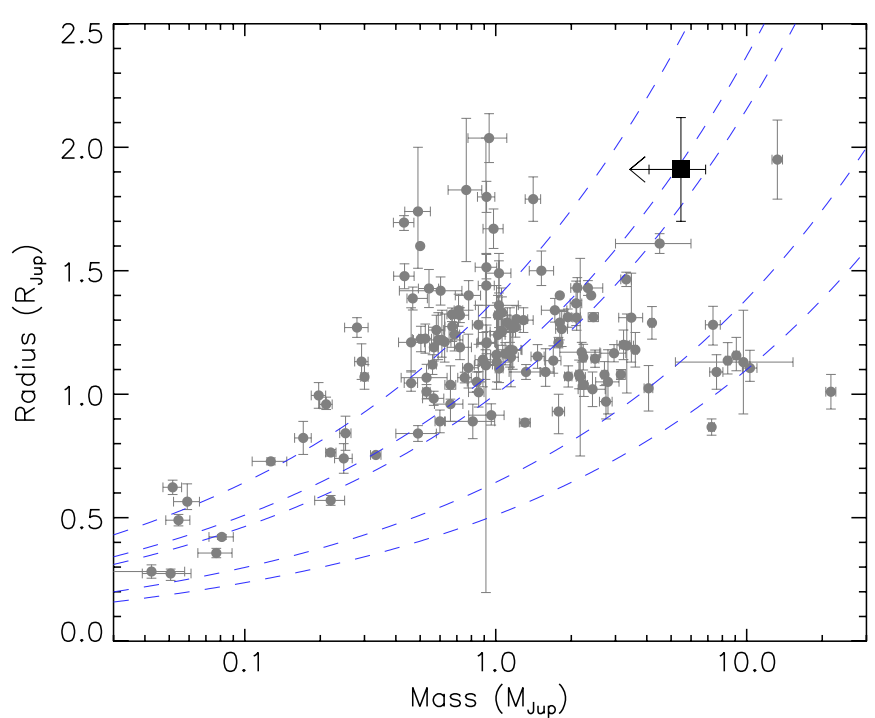

Figure 10. Radius vs. mass for the known transiting planets (Section 3.3; data taken from the NASA Exoplanet Archive). PTFO 8-8695b is marked as an upper mass limit, highlighted by the large square. Iso-density contours are marked at $0.5,1,1.33$ (Jupiter density), 5 , and $10 \mathrm{~g} \mathrm{~cm}^{-3}$.

(A color version of this figure is available in the online journal.)

Taken together, the measured $v_{\mathrm{r}} \sin i_{*}$ and the photometrically derived $v_{\mathrm{r}}$ give an estimated inclination of the stellar rotation axis, $i_{*}=42^{\circ} \pm 7^{\circ}$. Comparing this with the measured orbital inclination, $i_{\text {orb }}=61.8 \pm 3.7$, we see that there is weak evidence for a possible misalignment between the orbital and stellar rotation axes (consistent with the possibility of detecting changes in the orbital inclination on relatively short timescales, as mentioned in Section 3.1.2).

\subsection{Implications}

In Figure 10, we show the radius and mass of the companion in relation to the currently known transiting exoplanets listed in the NASA Exoplanet Archive, ${ }^{27}$ where we have indicated the mass of the candidate as an upper limit only. It clearly lies at the upper end of the gas-giant radius distribution, although not unprecedentedly so. A significantly inflated atmosphere is to be expected owing both to the system's very young age, and to stellar irradiation at the companion's exceptionally small orbital radius. Planetary atmosphere models are currently not well constrained in this regime due to the lack of known exoplanets that are both young and close-in. Marley et al. (2007) and Fortney et al. (2007) caution that evolutionary models should be treated with care at ages up to $10 \mathrm{Myr}$ or more, being highly dependent on initial conditions and the formation mechanism assumed. Indeed, Spiegel \& Burrows (2012) suggest that comparison of atmospheric models with observations of exoplanets at such young ages may be a good way of distinguishing between different formation scenarios. Both Spiegel \& Burrows (2012) and Marley et al. (2007) predict radii of around 1.6 $R_{\text {Jup }}$ for a $5 M_{\text {Jup }}$ planet at $3 \mathrm{Myr}$, for non-irradiated "hot-start" (gravitational collapse) model atmospheres; our error bars place our measured radius $\left(1.9 \pm 0.2 R_{\odot}\right)$ just above this value. Our radius lies substantially farther above the post-formation cold-start (core collapse) isochrones, though comparison with the latter models is confused by uncertainty in the formation timescale, which is likely comparable to the age of the star. Accounting for

\footnotetext{
27 http://exoplanetarchive.ipac.caltech.edu
}

stellar irradiation could increase the theoretical radius further. Increases of $\sim 10 \%$ or more are typical at $\sim 0.02 \mathrm{AU}$ from a solar like star (Baraffe et al. 2010; Chabrier et al. 2004), where the incident flux is similar to that on our planet candidate (which is closer in, but orbiting a lower-mass star). The companion's proximity to the Roche limit may also weaken the gravitational binding of the object, further increasing its expected radius. Various other explanations are also proposed to explain the excess "radius anomaly" that is increasingly found in other gas-giant exoplanets (e.g., Baraffe et al. 2010; Chabrier et al. 2011). Our estimated radius is therefore not unreasonable, but due to both the model and observation uncertainties, a robust comparison with theory is difficult.

The apparent companion to PTFO 8-8695 also orbits close enough to its parent star that the consequent small size of its Roche lobe may be relevant. Below a certain mass threshold, it may not have sufficient self-gravity to hold itself together, and thus may begin to lose mass. Following Faber et al. (2005) and Ford \& Rasio (2006), the Roche radius, $R_{\text {Roche }}$, according to Paczyński (1971), is given by

$$
\begin{aligned}
R_{\text {Roche }} & =0.462\left(M_{\mathrm{p}} / M_{*}\right)^{1 / 3} a \leqslant 0.462\left(M_{\mathrm{p}, \max } / M_{*}\right)^{1 / 3} a \\
& \approx 1.92 \pm 0.16
\end{aligned}
$$

where $M_{\mathrm{p} \text {,max }}$ is our previously estimated maximum mass for the companion. Comparing with the measured companion radius gives $R_{\mathrm{p}} / R_{\text {Roche }} \gtrsim 0.994 \pm 0.094$. Framing the argument another way, we can rewrite the Roche formula to estimate the Roche limiting orbital radius, $R_{\text {Roche }}$, in terms of the measured companion radius to find $a / a_{\text {Roche }} \lesssim 1.008 \pm 0.095$ at the most-consistent with being at or within the Roche limit, within the errors. The planet may be sufficiently inflated that it fills its Roche lobe, and consequently may have lost mass in the past, or be in the process of losing mass (thus maintaining itself at the Roche limit).

\section{CONCLUSIONS}

We have detected transits from a candidate young planet orbiting a previously identified corotating or near-corotating $\approx 2.7$ Myr old M3 WTTS. Although we cannot completely rule out a false positive due to source blends, we are able to rule out confusion at the level of $\Delta H \approx 4.3$ mag beyond a separation of 0.25 , and argue qualitatively that a false detection due to a blended eclipsing binary is unlikely. The companion is in an exceptionally rapid 0.448413 day orbit, placing it among the shortest of the currently known exoplanet periods (cf., Demory et al. 2011; Charpinet et al. 2011; Muirhead et al. 2012; Rappaport et al. 2012). With an orbital radius only around twice the stellar radius, it appears to be at or within its Roche limiting orbit, with $a / a_{\text {Roche }} \lesssim 1.01 \pm 0.10$, raising the possibility of past or ongoing evaporation and mass loss. Perhaps the companion has been migrating and losing any mass beyond its Roche lobe as it does so; or perhaps it is continually being inflated to fill its Roche lobe, with any material which overflows being stripped away.

Although the transit photometry and the RV data both phase fold on the same periods, there is an apparent offset in phase between them. The most likely explanation is that the RV signal is shifted or dominated by the effect of star spots; we therefore suggest an upper limit on the (inclination independent) companion mass of $\approx 5 M_{\text {Jup }}$ based on the amplitude of the RV modulation. If it can be assumed that the object has had time to reach a stable (or quasi-stable) state-i.e., that mass loss 
rates are not too rapid, and that there have been no recent dramatic changes in the orbital geometry-then Roche limit considerations would imply a lower limit of a similar order, since anything much less would be unable to gravitationally bind the material within the measured companion radius.

The data are complex enough that we cannot yet be certain of a planet detection. In favor of the planetary interpretation, however, we note that: (1) the photometric transit shape appears to be flat bottomed with sharp ingress and egress slopes, and is difficult to explain with a star-spot model alone; (2) the transits appear highly consistent and periodic over a period of $\gtrsim 1 \mathrm{yr}$, which would be unusual for spots; (3) the RV signal places an upper mass limit well within the planetary regime; (4) a false positive due to a faint blended eclipsing binary is argued against by two observations which are suggestive of a transiting object associated with the primary TTS observed: The stellar rotation rate appears very close to the transit period, suggesting corotation, and the photometric variation from transit to transit is consistent with the notion of a planet transiting a heavily spotted TTS.

Further spectroscopic and photometric observations are needed to provide a clearer picture. Since the star is such a fast rotator, the expected RM effect (hinted at in the data) may provide a valuable opportunity for confirmation of the candidate. Most RV noise sources will be constant in the timescale of one transit, so further RV observations during the transit window could potentially provide full sampling of the effect. This would help confirm the planetary interpretation of the data, and could provide further useful information on the system geometry. Revisiting the target to make further measurements of the RV phase with respect to the transit ephemeris would help confirm the spot-interference interpretation: If spots are significant, the phase offset is likely to change with time as the spots change. Infrared RV observations, which are less sensitive to spot-induced noise, would also provide valuable further information, perhaps allowing for an unambiguous determination of the companion mass.

Given the young age of the system, the planet candidate is likely hot, and so a secondary transit may also be detectable with more precise photometry, allowing constraints to be placed on the companion temperature. Finally, simultaneous multi-band photometry and spectroscopy (particularly in the infrared, where spot-effects should be lessened) could further help disentangle the signatures of star spots and companion. This would also help to establish the cause of the apparent overall change in transit shape between the two years' observing runs, which could result either from changes in the spot distribution or possibly, given the exceptionally short orbital timescale, changes in the orbital geometry.

If our interpretation of the data is correct, the putative planet's youth and its unique proximity to its host star will make it a valuable object for helping inform our understanding of exoplanet formation. Its young age could have important implications for the mechanism by which it formed: Conventional core-accretion formation models (Pollack et al. 1996) occur on timescales of 1-10 Myr, comparable with or longer than the age of the system; the much more rapid gravitational-instability mechanism (Boss 1997, 2000) occurs on timescales orders of magnitudes shorter, and may thus perhaps be favored (see Baraffe et al. 2010 for a brief overview and comparison of the two mechanisms). The companion's inflated atmosphere appears indicative of the "hot start" models of Marley et al. (2007) and Spiegel \& Burrows (2012), which are associated with gravitational in- stability. At the same time, we cannot rule out that formation may yet be incomplete given the young age of the system. Neither can we rule out that the companion may be on the verge of evaporation and may not survive the lifetime of the star. Indeed, very few large planets are known to orbit small stars, and this object may well be transient. Much ambiguity remains at these young ages, and more observation and analysis of the system is needed. If the planetary nature of the proposed companion is confirmed, the system could provide a wealth of new information for constraining dynamical and atmospheric evolution models for exoplanets. We therefore propose PTFO 8-8695 as an object worthy of further careful study by the community.

The authors acknowledge Tim Morton and Dimitri Veras for their helpful input. S.B.C. wishes to acknowledge generous support from Gary and Cynthia Bengier, the Richard and Rhoda Goldman Fund, National Aeronautics and Space Administration (NASA)/Swift grant NNX10AI21G, NASA/Fermi grant NNX1OA057G, and National Science Foundation (NSF) grant AST-0908886. S.M. acknowledges support from NSF awards AST-1006676, AST-1126413, PSARC, and the NASA Astrobiology Institute.

Observations obtained with the Samuel Oschin Telescope at the Palomar Observatory as part of the Palomar Transient Factory project, a scientific collaboration between the California Institute of Technology, Columbia University, Las Cumbres Observatory, the Lawrence Berkeley National Laboratory, the National Energy Research Scientific Computing Center, the University of Oxford, and the Weizmann Institute of Science.

The Hobby-Eberly Telescope (HET) is a joint project of the University of Texas at Austin, the Pennsylvania State University, Stanford University, Ludwig-Maximilians-Universität München, and Georg-August-Universität Göttingen. The HET is named in honor of its principal benefactors, William P. Hobby and Robert E. Eberly.

Some of the data presented herein were obtained at the W. M. Keck Observatory, which is operated as a scientific partnership among the California Institute of Technology, the University of California and the National Aeronautics and Space Administration. The Observatory was made possible by the generous financial support of the W. M. Keck Foundation. The authors wish to recognize and acknowledge the very significant cultural role and reverence that the summit of Mauna Kea has always had within the indigenous Hawaiian community. We are most fortunate to have the opportunity to conduct observations from this mountain.

The Byrne Observatory at Sedgwick (BOS) is operated by the Las Cumbres Observatory Global Telescope Network and is located at the Sedgwick Reserve, a part of the University of California Natural Reserve System.

This research has made use of the NASA Exoplanet Archive, which is operated by the California Institute of Technology, under contract with the National Aeronautics and Space Administration under the Exoplanet Exploration Program.

This publication makes use of data products from the Two Micron All Sky Survey, which is a joint project of the University of Massachusetts and the Infrared Processing and Analysis Center/California Institute of Technology, funded by the National Aeronautics and Space Administration and the National Science Foundation.

This research has made use of the VizieR catalog access tool, CDS, Strasbourg, France, and of the software package 
Uncertainties: a Python package for calculations with uncertainties, Eric O. Lebigot. ${ }^{28}$

This work was partially supported by funding from the Center for Exoplanets and Habitable Worlds. The Center for Exoplanets and Habitable Worlds is supported by the Pennsylvania State University, the Eberly College of Science, and the Pennsylvania Space Grant Consortium.

Support for this work was provided by an award issued by JPL/Caltech.

Facilities: PO:1.2m (PTF), HET (HRS), Keck:I (HIRES), Keck:II (NIRC2), LCOGT (BOS), FTN, FTS, Mayall, Hale

\section{REFERENCES}

Agüeros, M. A., Covey, K. R., Lemonias, J. J., et al. 2011, ApJ, 740, 110 Aigrain, S., Hodgkin, S., Irwin, J., et al. 2007, MNRAS, 375, 29

Armitage, P. J. 2009, in AIP Conf. Proc. 1192, XIII Special Courses at the National Observatory of Rio de Janeiro, ed. F. Roig, D. Lopes, R. de La Reza, \& V. Ortega (Melville, NY: AIP), 3

Baraffe, I., Chabrier, G., Allard, F., \& Hauschildt, P. H. 1998, A\&A, 337,403

Baraffe, I., Chabrier, G., \& Barman, T. 2010, Rep. Prog. Phys., 73, 016901

Bender, C. F., Mahadevan, S., Deshpande, R., et al. 2012, ApJ, 751, L31

Boss, A. P. 1997, Science, 276, 1836

Boss, A. P. 2000, ApJ, 536, L101

Briceño, C., Calvet, N., Hernández, J., et al. 2005, AJ, 129, 907

Briceño, C., Hartmann, L., Hernández, J., et al. 2007, ApJ, 661, 1119

Carter, J. A., \& Winn, J. N. 2009, ApJ, 704, 51

Chabrier, G., Barman, T., Baraffe, I., Allard, F., \& Hauschildt, P. H. 2004, ApJ, 603, L53

Chabrier, G., Leconte, J., \& Baraffe, I. 2011, in IAU Symp. 276, The Astrophysics of Planetary Systems: Formation, Structure, and Dynamical Evolution, ed. A. Sozzetti, M. G. Lattanzi, \& A. P. Boss (Cambridge: Cambridge Univ. Press), 171

Charpinet, S., Fontaine, G., Brassard, P., et al. 2011, Nature, 480, 496

Claret, A. 2000, A\&A, 363, 1081

Crockett, C. J., Mahmud, N. I., Prato, L., et al. 2011, ApJ, 735, 78

Dawson, R. I., \& Fabrycky, D. C. 2010, ApJ, 722, 937

Demory, B.-O., Gillon, M., Deming, D., et al. 2011, A\&A, 533, A114

Esposito, M., Guenther, E., Hatzes, A. P., \& Hartmann, M. 2006, in Tenth Anniversary of 51 Peg-b: Status of and Prospects for Hot Jupiter Studies, ed. L. Arnold, F. Bouchy, \& C. Moutou (Paris: Frontier Group), 127

Faber, J. A., Rasio, F. A., \& Willems, B. 2005, Icarus, 175, 248

Ford, E. B., \& Rasio, F. A. 2006, ApJ, 638, L45

Fortney, J. J., Marley, M. S., \& Barnes, J. W. 2007, ApJ, 659, 1661

Fortney, J. J., \& Nettelmann, N. 2010, Space Sci. Rev., 152, 423

Gaudi, B. S., \& Winn, J. N. 2007, ApJ, 655, 550

Gazak, J. Z., Johnson, J. A., Tonry, J., et al. 2012, Adv. Astron., 2012, 697967
Gray, D. F. 1992, The Observation and Analysis of Stellar Photospheres (2nd ed.; Cambridge: Cambridge Univ. Press)

Heacox, W. D. 1987, J. Opt. Soc. Am. A., 4, 488

Hernández, J., Calvet, N., Briceño, C., et al. 2007, ApJ, 671, 1784

Hillenbrand, L. A. 2008, Phys. Scr. T, 130, 014024

Howard, A. W., Johnson, J. A., Marcy, G. W., et al. 2009, ApJ, 696, 75

Huerta, M., Johns-Krull, C. M., Prato, L., Hartigan, P., \& Jaffe, D. T. 2008, ApJ, 678,472

Kraus, A. L., \& Ireland, M. J. 2012, ApJ, 745, 5

Kurucz, R. L. 2005, Mem. Soc. Astron. Ital. Suppl., 8, 14

Law, N. M., Dekany, R. G., Rahmer, G., et al. 2010, Proc. SPIE, 7735, 77353M

Law, N. M., Kulkarni, S. R., Dekany, R. G., et al. 2009, PASP, 121, 1395

Levitan, D., Fulton, B. J., Groot, P. J., et al. 2011, ApJ, 739, 68

Lomb, N. R. 1976, Ap\&SS, 39, 447

Mahmud, N. I., Crockett, C. J., Johns-Krull, C. M., et al. 2011, ApJ, 736, 123

Mandel, K., \& Agol, E. 2002, ApJ, 580, L171

Marley, M. S., Fortney, J. J., Hubickyj, O., Bodenheimer, P., \& Lissauer, J. J. 2007, ApJ, 655, 541

Marsh, K. A., Kirkpatrick, J. D., \& Plavchan, P. 2010, ApJ, 709, L158

McLaughlin, D. B. 1924, ApJ, 60, 22

Miller, A. A., Irwin, J., Aigrain, S., Hodgkin, S., \& Hebb, L. 2008, MNRAS, 387, 349

Miralda-Escudé, J. 2002, ApJ, 564, 1019

Muirhead, P. S., Johnson, J. A., Apps, K., et al. 2012, ApJ, 747, 144

Neuhäuser, R., Errmann, R., Berndt, A., et al. 2011, Astron. Nachr., 332, 547

Nguyen, D. C., Brandeker, A., van Kerkwijk, M. H., \& Jayawardhana, R. 2012, ApJ, 745, 119

Ofek, E. O., Laher, R., Law, N., et al. 2012, PASP, 124, 62

Paczyński, B. 1971, ARA\&A, 9, 183

Paulson, D. B., \& Yelda, S. 2006, PASP, 118, 706

Plavchan, P., Jura, M., Kirkpatrick, J. D., Cutri, R. M., \& Gallagher, S. C. 2008, ApJS, 175, 191

Pollack, J. B., Hubickyj, O., Bodenheimer, P., et al. 1996, Icarus, 124, 62

Prato, L., Huerta, M., Johns-Krull, C. M., et al. 2008, ApJ, 687, L103

Ramsey, L. W., Adams, M. T., Barnes, T. G., et al. 1998, Proc. SPIE, 3352, 34

Rappaport, S., Levine, A., Chiang, E., et al. 2012, ApJ, 752, 1

Rau, A., Kulkarni, S. R., Law, N. M., et al. 2009, PASP, 121, 1334

Rossiter, R. A. 1924, ApJ, 60, 15

Scargle, J. D. 1982, ApJ, 263, 835

Schneider, J., Dedieu, C., Le Sidaner, P., Savalle, R., \& Zolotukhin, I. 2011, A\&A, 532, A79

Setiawan, J., Henning, T., Launhardt, R., et al. 2008, Nature, 451, 38

Setiawan, J., Weise, P., Henning, T., et al. 2007, ApJ, 660, L145

Shetrone, M., Cornell, M. E., Fowler, J. R., et al. 2007, PASP, 119, 556

Siess, L., Dufour, E., \& Forestini, M. 2000, A\&A, 358, 593

Skrutskie, M. F., Cutri, R. M., Stiening, R., et al. 2006, AJ, 131, 1163

Spiegel, D. S., \& Burrows, A. 2012, ApJ, 745, 174

Tull, R. G. 1998, Proc. SPIE, 3355, 387

van Eyken, J. C., Ciardi, D. R., Rebull, L. M., et al. 2011, AJ, 142, 60

Vogt, S. S., Allen, S. L., Bigelow, B. C., et al. 1994, Proc. SPIE, 2198, 362

Wright, J. T., \& Howard, A. W. 2009, ApJS, 182, 205

28 http://packages.python.org/uncertainties 